Pacific Journal of Mathematics

ON DISPERSIVE OPERATORS IN BANACH LATTICES 


\title{
ON DISPERSIVE OPERATORS IN BANACH LATTICES
}

\section{Ken-ITi SATo}

\begin{abstract}
Dispersive operators were introduced by $R$. S. Phillips for characterization of infinitesimal generators of nonnegative contraction semigroups in Banach lattices. Later other definitions of dispersiveness were given by $M$. Hasegawa and $K$. Sato. H. Kunita, for the purpose of application to Markov processes, introduced the notion of complete $\gamma$-dispersiveness which characterizes the infinitesimal generators of $e$-majoration preserving nonnegative semigroups $T_{t}$ with norm $\leqq e^{\gamma t}$. In this paper we will give a unified treatment of these results. Further, we will clarify the relation between dispersiveness and dissipativeness in some cases. We consider also characterization of infinitesimal generators of nonnegative semigroups without norm conditions.
\end{abstract}

Let $\mathfrak{B}$ be a Banach lattice. That $1 \mathrm{~s}, \mathfrak{B}$ is a vector lattice and a real Banach space at the same time and $|f| \leqq|g|$ implies $\|f\| \leqq\|g\|$. We use the notations $f^{+}=f \vee 0, f^{-}=-(f \wedge 0)$, and $|f|=f \vee(-f)$. Following Kunita [8], let $\widetilde{\mathfrak{B}}$ be a vector lattice which is an extension of $\mathfrak{B}$, and let $e$ be an element of $\widetilde{\mathfrak{B}}$. We say that an operator $T$ is e-majoration preserving if $f \leqq e$ implies $T f \leqq e$. Let $\boldsymbol{G}$ be the set of infinitesimal generators of strongly continuous semigroups of linear operators in $\mathfrak{B}$. For real numbers $M \geqq 1$ and $\gamma$, let $\boldsymbol{G}(M, \gamma)$ be the set of $A \in G$ such that the generated semigroup $T_{t}$ satisfies $\left\|T_{t}\right\| \leqq M e^{\gamma t}$, $G^{e}$ be the set of $A \in G$ such that $T_{t}$ is $e$-majoration preserving, and further, let $\boldsymbol{G}^{e}(M, \gamma)=\boldsymbol{G}(M, \gamma) \cap \boldsymbol{G}^{e}$. For linear operators, 0-majoration preserving is the same as nonnegativity and $\boldsymbol{G}^{0}$ is denoted by $\boldsymbol{G}^{+}$. We assume that $e$ satisfies

$$
f \in \mathfrak{B} \text { implies } f \wedge e \in \mathfrak{B} \text {; }
$$

$$
f \wedge \alpha e \text { converges weakly to } f \wedge 0 \text { as } \alpha \rightarrow 0+\text { for each } f \in \mathfrak{B} \text {; }
$$

$$
e \geqq 0 \text {. }
$$

Note that $f \wedge \alpha e \in \mathfrak{B}$ for $\alpha>0$ by (0.1). We call a real-valued functional $\psi_{e}(f, g)$ on $\mathfrak{B} \times \mathfrak{B}$ e-gauge functional, if the following are satisfied:

$$
\begin{aligned}
& \text { If } g \leqq e \text { and } \alpha>0 \text { then } \psi_{e}(f, \alpha(f \wedge e-g)) \geqq 0 \text { and } \\
& \qquad \psi_{e}(f, \alpha(g-f \wedge e)) \leqq 0 ; \\
& \psi_{e}(f, g+h) \leqq\|g\|+\psi_{e}(f, h) ;
\end{aligned}
$$


$(0.6)$

$$
\psi_{e}\left(f, \alpha(f-e)^{+}+g\right)=\alpha\left\|(f-e)^{+}\right\|+\psi_{e}(f, g) \text { for all } \alpha \text {. }
$$

Note that $(f-e)^{+}=f-f \wedge e \in \mathfrak{B}$ for $f \in \mathfrak{B}$. Let $\gamma$ be a real number. We call an operator $A\left(\psi_{e}, \gamma\right)$-dispersive if

$$
\psi_{e}(f, A f) \leqq \gamma\left\|(f-e)^{+}\right\| \text {whenever }(f-e)^{+} \neq 0 .
$$

For any $e$-gauge functional $\psi_{e}$, we will prove the following:

ThEOREM 1.1. If $A \in G^{e}(1, \gamma)$, then $A$ is $\left(\psi_{e}, \gamma\right)$-dispersive.

THEOREM 1.2. If $A$ is a densely defined ( $\left.\psi_{e}, \gamma\right)$-dispersive operator with $\mathfrak{R}(\alpha-A)=\mathfrak{B}$ for some $\alpha>\gamma$, then $A \in G^{e}(1, \gamma)$.

These theorems include the results by Phillips [10], Hasegawa [5], and Sato [11] on characterization of the operators in $G^{+}(1,0)$ and Kunita's result [8] on $\boldsymbol{G}^{e}(1, \gamma) \cap \boldsymbol{G}^{+}$, except that Kunita does not assume (0.2). (See Remark 1.3 concerning this point.)

In $\S 1$, we will prove the above theorems. In $\S 2$, existence and further properties of $e$-gauge functionals will be discussed. In particular, we introduce new functionals $\varphi_{e}$ and $\varphi_{e}^{\prime}$ and prove that they are the maximum and the minimum $e$-gauge functionals. More examples of $e$-gauge functionals are found in $\S 3$. They include various functionals used in definition of dispersive operators by Phillips, Hasegawa, and Sato, and of completely $\gamma$-dispersive operators by Kunita. In $\S 4$, we will give remarks related with the closability of $\left(\psi_{e}, \gamma\right)$ dispersive operators. Some results on the relation between dispersiveness and dissipativeness will be given in $\S 5$. In $\S 6$ we will discuss a necessary condition for an operator to belong to $G^{+}$and prove that this is also sufficient in some special cases.

The author thanks Hiroshi Kunita for informing him of his work that was to appear in [8].

\section{Characterization of $G^{e}(1, \gamma)$.}

THEOREM 1.1. Suppose that e satisfies (0.1)-(0.3) and let $\psi_{e}$ be an e-gauge functional. Then, any operator in $G^{e}(1, \gamma)$ is $\left(\psi_{e}, \gamma\right)$-dispersive.

Proof. Let $T_{t}$ be the semigroup generated by $A \in G^{e}(1, \gamma)$ and let $f \in \mathfrak{D}(A)$. We have

$$
\begin{aligned}
\psi_{e}\left(f, t^{-1}\left(T_{t} f-f\right)\right) \leqq & t^{-1} e^{\gamma t}\left\|T_{t}(f-e)^{+}\right\|-t^{-1}\left\|(f-e)^{+}\right\| \\
& +\psi_{e}\left(f, t^{-1}\left[\left(1-e^{-\gamma t}\right) T_{t}(f-e)^{+}\right.\right. \\
& \left.\left.+T_{t}(f \wedge e)-f \wedge e\right]\right) \\
\leqq & \psi_{e}\left(f, t^{-1}\left[\left(1-e^{-\gamma t}\right) T_{t}(f-e)^{+}\right.\right. \\
& \left.\left.+T_{t}(f \wedge e)-f \wedge e\right]\right)
\end{aligned}
$$


by $(0.5),(0.6)$, and $\left\|T_{t}\right\| \leqq e^{-\gamma t}$. The last member is not greater than

$$
\begin{aligned}
& \left\|t^{-1}\left(1-e^{-\gamma t}\right) T_{t}(f-e)^{+}-\gamma(f-e)^{+}\right\| \\
& \quad+\gamma\left\|(f-e)^{+}\right\|+\psi_{e}\left(f, t^{-1}\left[T_{t}(f \wedge e)-f \wedge e\right]\right)
\end{aligned}
$$

by $(0.5)$ and $(0.6)$. Noting that the last term is not positive by (0.4) since $T_{t}(f \wedge e) \leqq e$, and that the first term tends to zero as $t \rightarrow 0+$, we get

$$
\psi_{e}(f, A f)=\lim _{t \rightarrow 0+} \psi_{e}\left(f, t^{-1}\left(T_{t} f-f\right)\right) \leqq \gamma\left\|(f-e)^{+}\right\| \cdot
$$

Notice that any $e$-gauge functional $\psi_{e}(f, g)$ is continuous in $g$, because (0.5) implies

$$
\left|\psi_{e}(f, g)-\psi_{e}(f, h)\right| \leqq\|g-h\| \text {. }
$$

The proof of Theorem 1.1 is complete.

REMARK 1.1. Above we have proved more than $\left(\psi_{e}, \gamma\right)$-dispersiveness: $\psi_{e}(f, A f) \leqq \gamma\left\|(f-e)^{+}\right\|$for all $f \in \mathfrak{D}(A)$.

Let us prepare lemmas for the proof of Theorem 1.2. For elementary properties of vector lattices, we refer to Birkhoff [1] or Yosida [12].

LEMma 1.1. If $e$ is an element of $\widetilde{\mathfrak{B}}$ satisfying (0.1) and $\psi_{e}$ is an e-gauge functional, then

$$
\psi_{e}(f, g) \geqq \alpha\left\|(f-e)^{+}\right\|-\left\|(\alpha(f-e)-g)^{+}\right\|, \quad \alpha \geqq 0 .
$$

Proof. We may assume $\alpha>0$. We have, by (0.5) and (0.6),

$$
\begin{aligned}
\psi_{e}(f, g)= & \alpha\left\|(f-e)^{+}\right\|+\psi_{e}\left(f, g-\alpha(f-e)-\alpha(f-e)^{-}\right) \\
\geqq & \alpha\left\|(f-e)^{+}\right\|-\left\|(\alpha(f-e)-g)^{+}\right\| \\
& +\psi_{e}\left(f,(g-\alpha(f-e))^{+}-\alpha(f-e)^{-}\right) .
\end{aligned}
$$

The last term is $\psi_{e}\left(f, \alpha\left[\left(\alpha^{-1} g-f+e\right)^{+}-e+f \wedge e\right]\right)$ and hence, is nonnegative by $(0.4)$.

Lemma 1.2. Suppose e satisfies (0.1) - (0.3). If $A$ is linear and $\left(\psi_{e}, \gamma\right)$-dispersive in some e-gauge functional $\psi_{e}$, then

$$
\begin{array}{cc}
(\alpha-\gamma)\left\|(f-e)^{+}\right\| \leqq\left\|(\alpha(f-e)-A f)^{+}\right\| & \text {for } \alpha \geqq 0, \\
(\alpha-\gamma)\left\|f^{+}\right\| \leqq\left\|(\alpha f-A f)^{+}\right\| & \text {for all } \alpha, \\
(\alpha-\gamma)\|f\| \leqq 2\|\alpha f-A f\| & \text { for all } \alpha .
\end{array}
$$


Proof. (1.3) is a direct consequence of (0.7) and (1.2). Hence we have, for $\alpha \geqq 0$ and $\varepsilon>0$,

$$
(\alpha-\gamma)\left\|(f-\varepsilon e)^{+}\right\| \leqq\left\|(\alpha(f-\varepsilon e)-A f)^{+}\right\| \leqq\left\|(\alpha f-A f)^{+}\right\|,
$$

making use of (0.3). Since (0.2) implies $\liminf _{\varepsilon \rightarrow 0+}\left\|(f-\varepsilon e)^{+}\right\| \geqq\left\|f^{+}\right\|$, we have (1.4) for $\alpha \geqq 0$. For every $f$ and $\stackrel{\varepsilon \rightarrow 0+}{g}$ in $\mathfrak{B}$, let

$$
\begin{aligned}
\varphi(f, g) & =\lim _{\varepsilon \rightarrow 0+} \varepsilon^{-1}\left(\left\|(f+\varepsilon g)^{+}\right\|-\left\|f^{+}\right\|\right) \\
& =\lim _{\alpha \rightarrow \infty}\left(\left\|(\alpha f+g)^{+}\right\|-\left\|\alpha f^{+}\right\|\right) .
\end{aligned}
$$

The limit exists and is finite, since $\left\|(f+\varepsilon g)^{+}\right\|$is a convex function of $\varepsilon$. We have $\varphi(f, g) \leqq\left\|g^{+}\right\|$since $(f+\varepsilon g)^{+} \leqq f^{+}+\varepsilon g^{+}, \varepsilon>0$. Also, we have $\varphi(f, \alpha f+g)=\alpha\left\|f^{+}\right\|+\varphi(f, g)$ for all $\alpha$, which is easily checked. (1.4) for large $\alpha$ implies $\varphi(f,-A f) \geqq-\gamma\left\|f^{+}\right\|$. Thus we get

$$
\begin{aligned}
\left\|(\alpha f-A f)^{+}\right\| & \geqq \varphi(f, \alpha f-A f) \\
& =\alpha\left\|f^{+}\right\|+\varphi(f,-A f) \geqq(\alpha-\gamma)\left\|f^{+}\right\|
\end{aligned}
$$

for all $\alpha$. (1.5) follows from (1.4) by

$$
\max \left\{\left\|f^{+}\right\|,\left\|f^{-}\right\|\right\} \leqq\|f\| \leqq\left\|f^{+}\right\|+\left\|f^{-}\right\| \text {. }
$$

Lemma 1.3. Let $A$ be linear and suppose that there exist real numbers $M>0$ and $\gamma$ such that

$$
(\alpha-\gamma)\|f\| \leqq M\|\alpha f-A f\| \text { for } f \in \mathfrak{D}(A), \alpha>\gamma .
$$

If $\mathfrak{R}(\alpha-A)=\mathfrak{B}$ for some $\alpha>\gamma$, then the same is true for every $\alpha>\gamma$.

Proof. Let $\alpha_{0}>\gamma$ and $\mathfrak{R}\left(\alpha_{0}-A\right)=\mathfrak{B}$. Then $\left(\alpha_{0}-A\right)^{-1}$ exists on $\mathfrak{B}$. Given $\alpha$ and $g$, define an operator $P$ by

$$
P u=\left(\alpha_{0}-A\right)^{-1}\left(g+\left(\alpha_{0}-\alpha\right) u\right), \quad u \in \mathfrak{B} .
$$

If $u$ is a fixed point for $P$, then $u$ satisfies $(\alpha-A) u=g$. But, $P$ has a fixed point whenever $\left|\alpha-\alpha_{0}\right|<\left(\alpha_{0}-\gamma\right) / M$, since we have

$$
\begin{aligned}
\|P u-P v\| & =\left\|\left(\alpha_{0}-A\right)^{-1}\left(\alpha_{0}-\alpha\right)(u-v)\right\| \\
& \leqq M\left(\alpha_{0}-\gamma\right)^{-1}\left|\alpha_{0}-\alpha\right|\|u-v\| .
\end{aligned}
$$

Hence, $\Re(\alpha-A)=\mathfrak{B}$ is proved for all $\alpha>\gamma$. This proof is due to Kömura [7] and can be applied to nonlinear case.

Lemma 1.4. Let $A$ be linear. If $\Re(\alpha-A)$ is a sublattice for $\alpha>\gamma$ and (1.4) holds, for $f \in \mathfrak{D}(A)$, then 


$$
(\alpha-\gamma)\|f\| \leqq\|\alpha f-A f\| \quad \text { for all } \alpha \text {. }
$$

Proof. We may assume $\alpha>\gamma$. We get

$$
(\alpha-\gamma)\left\|f^{-}\right\| \leqq\left\|(\alpha f-A f)^{-}\right\|
$$

together with (1.4). Hence $\alpha-A$ is one-to-one and $G_{\alpha}=(\alpha-A)^{-1}$ is nonnegative. Since

$$
\left|G_{\alpha} g\right| \leqq\left|G_{\alpha} g^{+}\right|+\left|G_{\alpha} g^{-}\right|=G_{\alpha} g^{+}+G_{\alpha} g^{-}=G_{\alpha}|g|,
$$

(1.7) follows from (1.4).

LEMma 1.5. Let $e$ be an element satisfying (0.1). If $A \in \boldsymbol{G}$ and if $\alpha G_{\alpha}=\alpha(\alpha-A)^{-1}$ is e-majoration preserving for all large $\alpha$, then $A \in \boldsymbol{G}^{e}$.

Proof. In general, if $f_{n} \in \mathfrak{B}, f_{n} \leqq e$, and $f_{n} \rightarrow f$ strongly, then $f_{n} \wedge e \rightarrow f \wedge e$ strongly and $f \leqq e$. Let $g \in \mathfrak{B}$ and $g \leqq e$. Then, $\left(\alpha G_{\alpha}\right)^{n} g \leqq e$ and $e^{t \alpha^{2} G_{\alpha}} g \leqq e^{t \alpha} e$. Hence $T_{t} g=\lim _{\alpha \rightarrow \infty} e^{-t \alpha} e^{t \alpha^{2} G_{\alpha}} g \leqq e$.

Now we can prove the following

THEOREM 1.2. Let e satisfy (0.1)-(0.3) and let $\psi_{e}$ be an e-gauge functional. If $A$ is a densely defined $\left(\psi_{e}, \gamma\right)$-dispersive linear operator with $\Re(\alpha-A)=\mathfrak{B}$ for some $\alpha>\gamma$, then $A \in G^{e}(1, \gamma)$.

Proof. By Lemmas 1.2 and 1.3, we have $\mathfrak{R}(\alpha-A)=\mathfrak{B}$ for all $\alpha>\gamma$. Hence we have (1.7) for all $\alpha>\gamma$ by Lemma 1.4. It follows from the Hille-Yosida theorem that $A \in \boldsymbol{G}(1, \gamma)$. For any $\alpha>\max \{\gamma, 0\}$, let us prove that $\alpha G_{\alpha}=\alpha(\alpha-A)^{-1}$ is $e$-majoration preserving. If $\alpha G_{\alpha} g=u$ and $g \leqq e$, then $(\alpha-\gamma)\left\|(u-e)^{+}\right\| \leqq \alpha\left\|(g-e)^{+}\right\|=0$ by (1.3), and hence $u \leqq e$. Thus $A \in G^{e}(1, \gamma)$ by Lemma 1.5 and the proof is complete.

REMARK 1.2. If $e$ satisfies (0.1)-(0.3), every $e$-majoration preserving linear operator in $\mathfrak{B}$ is nonnegative. As a consequence, we have $\boldsymbol{G}^{e} \subset \boldsymbol{G}^{+}$. In fact, let $f \leqq 0$. For every $\alpha>0$ we have $f \leqq \alpha e$ by (0.3), and hence $T f \leqq \alpha e$. For any nonnegative $\varphi \in \mathfrak{B}^{*}$ we have $\lim _{\alpha \rightarrow 0+} \varphi(T f \wedge \alpha e)=\varphi(T f \wedge 0) \leqq 0$ by $(0.2)$, and hence $\varphi(T f) \leqq 0$. This means $T f \leqq 0$.

REMARK 1.3. If $e$ satisfies only (0.1), Theorem 1.1 holds true without any change, and the following theorem replaces Theorem 1.2: Let $A$ be a densely defined linear operator with $\Re(\alpha-A)=\mathfrak{B}$ for some $\alpha>\gamma$. If $A$ is $\left(\psi_{e}, \gamma\right)$-dispersive and $\left(\psi_{0}, \gamma\right)$-dispersive in some e-gauge functional $\psi_{e}$ and 0-gauge functional $\psi_{0}$, then 
$A \in G^{e}(1, \gamma) \cap \boldsymbol{G}^{+}$. The proof is carried out in the same way. This includes Kunita's result in [8], who assumes also (0.3) and $\gamma \geqq 0$ and uses the functional $\sigma$ (see $\S 3$ ) for gauge functional.

ExAMPLE 1.1. If $\widetilde{\mathfrak{B}}=\mathfrak{B}$, then any nonnegative $e$ satisfies $(0.1)-$ (0.3). In case $e=0$, the above theorems characterize the operators in $\boldsymbol{G}^{+}(1,0)$.

2. Functional $\varphi_{e}$. In this section, only the condition $(0.1)$ is assumed for $e$. We denote by $\mathfrak{B}^{e}$ the linear subspace of $\widetilde{\mathfrak{B}}$ spanned by $\mathfrak{B}$ and $e$. Let us define

$$
\begin{gathered}
\varphi_{e}(f, g)=\lim _{\varepsilon \rightarrow 0+} \varepsilon^{-1}\left(\left\|(f-e+\varepsilon g)^{+}\right\|-\left\|(f-e)^{+}\right\|\right) \\
=\lim _{\alpha \rightarrow \infty}\left(\left\|(\alpha(f-e)+g)^{+}\right\|-\left\|\alpha(f-e)^{+}\right\|\right) \\
\varphi_{e}^{\prime}(f, g)=-\varphi_{e}(f,-g) .
\end{gathered}
$$

Lemma 2.1. The limit in (2.1) exists and is finite for each $f \in \mathfrak{B}$ and $g \in \mathfrak{B}^{e}$.

Proof. Note that $\left\|(f-e+\varepsilon g)^{+}\right\|$is a convex function of $\varepsilon$ in a neighborhood of 0 .

Main results in this section are the following two theorems.

THEOREM 2.1. $\varphi_{e}(f, g)$ and $\varphi_{e}^{\prime}(f, g)$ are e-gauge functionals of $f$ and $g \in \mathfrak{B}$.

THEOREM 2.2. If $\psi_{e}$ is an e-gauge functional, then

$$
\varphi_{e}^{\prime}(f, g) \leqq \psi_{e}(f, g) \leqq \varphi_{e}(f, g), \quad f, g \in \mathfrak{B} \text {. }
$$

For the proof, we prepare some properties of $\varphi_{e}$.

Lemma 2.2. Let $f \in \mathfrak{B}, g, h \in \mathfrak{B}^{e}$, and $k \in \mathfrak{B}$. Then,

$$
\begin{array}{cr}
\varphi_{e}(f, 0)=0 ; & \\
\varphi_{e}(f, k-\alpha e) \leqq\left\|(k-\alpha e)^{+}\right\|, & \alpha \geqq 0 ; \\
\varphi_{e}(f, \alpha(f-e)+g)=\alpha\left\|(f-e)^{+}\right\|+\varphi_{e}(f, g), & \alpha \text { real } ; \\
\varphi_{e}(f, g+h) \leqq \varphi_{e}(f, g)+\varphi_{e}(f, h) ; & \\
g \leqq h \text { implies } \varphi_{e}(f, g) \leqq \varphi_{e}(f, h) ; & \\
\varphi_{e}\left(f, \alpha(f-e)^{-}+k\right)=\varphi_{e}(f, k), & \alpha \text { real } .
\end{array}
$$


Proof. (2.4)-(2.7) are proved easily from the definition and the property $(g+h)^{+} \leqq g^{+}+h^{+} . \quad(2.8)$ is evident since $g \leqq h$ implies $(f-e+\varepsilon g)^{+} \leqq(f-e+\varepsilon h)^{+}$. In order to prove (2.9), we may assume $\alpha>0$. Let $l=f-e$. Suppose, for a moment,

$$
\left(l+\varepsilon \alpha l^{-}+\varepsilon k\right)^{+} \leqq\left(l+\varepsilon(1-\varepsilon \alpha)^{-1} k\right)^{+} \vee(l+\varepsilon k)^{+}
$$

for sufficiently small $\varepsilon>0$. Since we have

$$
\begin{aligned}
& \left\|\left(l+\varepsilon(1-\varepsilon \alpha)^{-1} k\right)^{+} \vee(l+\varepsilon k)^{+}\right\|-\left\|(l+\varepsilon k)^{+}\right\| \\
\leqq & \left\|\left(l+\varepsilon(1-\varepsilon \alpha)^{-1} k\right)^{+}-(l+\varepsilon k)^{+}\right\| \leqq\left\|\varepsilon(1-\varepsilon \alpha)^{-1} k-\varepsilon k\right\| \\
= & \varepsilon^{2} \alpha(1-\varepsilon \alpha)^{-1}\|k\|,
\end{aligned}
$$

it follows from (2.10) that

$$
\begin{aligned}
\varepsilon^{-1}\left(\left\|\left(l+\varepsilon \alpha l^{-}+\varepsilon k\right)^{+}\right\|-\left\|l^{+}\right\|\right) \leqq & \varepsilon^{-1}\left(\left\|(l+\varepsilon k)^{+}\right\|-\left\|l^{+}\right\|\right) \\
& +\varepsilon \alpha(1-\varepsilon \alpha)^{-1}\|k\| .
\end{aligned}
$$

This implies $\varphi_{e}\left(f, \alpha l^{-}+k\right) \leqq \varphi_{e}(f, k)$. On the other hand, the reverse inequality is a consequence of (2.8) by $\alpha>0$. In order to prove (2.10), it suffices to show

$$
\begin{aligned}
\left(l+\beta l^{-}+h\right)^{+} \leqq & \left(l+(1-\beta)^{-1} h\right)^{+} \vee(l+h)^{+} \text {for } 0<\beta<1, \\
& l, h \in \widetilde{\mathfrak{B}} .
\end{aligned}
$$

Let $\gamma=\beta(1-\beta)^{-1}$. Since

$$
\begin{aligned}
& (\gamma h) \vee(-l-h) \vee 0+\beta l \\
= & (\gamma(h+l-\beta l)) \vee(-h-l+\beta l) \vee(\beta l) \geqq 0,
\end{aligned}
$$

we have

$$
\begin{aligned}
& \left(l+(1-\beta)^{-1} h\right) \vee(l+h) \vee 0-\left(l+\beta l^{-}+h\right) \\
= & (\gamma h) \vee(-l-h) \vee 0-\beta l^{-} \geqq 0 .
\end{aligned}
$$

This proves (2.11).

Proof of Theorem 2.1. Let us check (0.4)-(0.6) for $\varphi_{e}$. If $g \leqq e$ and $\alpha>0$, then

$$
\begin{aligned}
\varphi_{e}(f, \alpha(f \wedge e-g)) & \geqq \varphi_{e}(f, \alpha(f \wedge e-e)) \\
& =\varphi_{e}\left(f,-\alpha(f-e)^{-}\right)=0
\end{aligned}
$$

by (2.4), (2.8), and (2.9). The second inequality in (0.4) is proved similarly. (0.5) is a consequence of (2.5) and (2.7). (0.6) is obtained from (2.6) and (2.9). Hence $\varphi_{e}$ is an $e$-gauge functional. $\varphi_{e}^{\prime}$ is also an $e$-gauge functional by the next lemma. 
LEMma 2.3. If $\psi_{e}$ is an e-gauge functional and if $\psi_{e}^{\prime}$ is defined by

$$
\psi_{e}^{\prime}(f, g)=-\psi_{e}(f,-g),
$$

then $\psi_{e}^{\prime}$ is also an e-gauge functional.

Proof is trivial.

Proof of Theorem 2.2. The first inequality in (2.3) is obtained from Lemma 1.1. This implies the second inequality by virtue of Lemma 2.3.

REMARK 2.1. Theorems 2.1 and 2.2 imply the following assertion: Even if $\psi_{e}$ is not an e-gauge functional, Theorems 1.1 and 1.2 remain true provided that $\psi_{e}$ satisfies (2.3). In particular, (2.3) holds if $\psi_{e}$ satisfies $(0.4),(0.6)$, and

$$
\psi_{e}(f, g+h) \leqq\|g\|+\psi_{e}(f, h) \quad \text { for } g \geqq 0 \text {. }
$$

REMARK 2.2. One remarkable feature of $\varphi_{e}^{\prime}$ is this: An operator $A$ is $\left(\varphi_{e}^{\prime}, \gamma\right)$-dispersive if and only if (1.3) holds for every $f \in \mathfrak{D}(A)$ and large $\alpha$. As a consequence, if $A$ is closable and $\left(\psi_{e}, \gamma\right)$-dispersive: in some $\psi_{e}$ satisfying (2.3), then the closure $\bar{A}$ is $\left(\varphi_{e}^{\prime}, \gamma\right)$-dispersive.

REMARK 2.3. We list some more properties of $\varphi_{e}$ : Let $f \in \mathfrak{B}$, $g \in \mathfrak{B}^{e}$, and $k \in \mathfrak{B}$.

$$
\begin{array}{crr}
\varphi_{e}(f, k+\alpha e) \geqq-\left\|(k+\alpha e)^{-}\right\|, & \alpha \geqq 0 ; \\
\varphi_{e}(f, \alpha g)=\alpha \varphi_{e}(f, g), & \alpha \geqq 0 ; \\
\varphi_{\alpha e}(\alpha f, g)=\varphi_{e}(f, g), & \alpha>0 ; \\
\varphi_{e}^{\prime}(f, g) \leqq 0 \leqq \varphi_{e}(f, g) & \text { if }(f-e)^{+}=0 ; \\
\tau^{\prime}\left(f^{+}, k\right) \leqq \varphi_{0}^{\prime}(f, k) \leqq \varphi_{0}(f, k) \leqq \tau\left(f^{+}, k\right), &
\end{array}
$$

where we define

$$
\tau(f, k)=\lim _{\varepsilon \rightarrow 0+} \varepsilon^{-1}(\|f+\varepsilon k\|-\|f\|), \quad \tau^{\prime}(f, k)=-\tau(f,-k) .
$$

(2.13)-(2.16) are proved directly from the definition. For the proof of (2.17), notice that $(f+\varepsilon k)^{+} \leqq\left(f^{+}+\varepsilon k\right)^{+} \leqq\left|f^{+}+\varepsilon k\right| \cdot \quad(2.4)-(2.9)$ and (2.13)-(2.17) combined with Theorem 2.2 lead to many properties. of $e$-gauge functionals.

Simple examples of $\widetilde{\mathfrak{B}}, e$, and $\varphi_{e}$ are given in the following. 
These are proved in the same way as $[11, \S 6]$.

EXAmple 2.1. Let $\mathfrak{B}=\widetilde{\mathfrak{B}}=C(X)$, the space of continuous functions on a compact space $X$. Then,

$$
\begin{aligned}
\varphi_{e}(f, g) & =\max _{x:(f-e)(x)=1 \mid(f-e)+1 !} g(x) \text { if }(f-e)^{+} \neq 0, \\
& =\max _{x:(f-e)(x)=0} g^{+}(x) \text { if } f-e \leqq 0 \text { and }(f-e)(x)=0 \text { for some } x, \\
& =0 \\
& \text { if }(f-e)(x)<0 \text { for all } x .
\end{aligned}
$$

As another example, let $X$ be a locally compact space which is not compact, $\mathfrak{B}$ be the space $C_{0}(X)$ of continuous functions on $X$ vanishing at infinity, and $\widetilde{\mathfrak{B}}$ be the vector lattice of all continuous functions on $X$. Then, any nonnegative $e$ in $\widetilde{\mathfrak{B}}$ satisfies $(0.1)-(0.3)$, and $\varphi_{e}$ has the same expression as above.

EXAMPLE 2.2. Let $(X, \mathscr{B}, m)$ be a measure space, $\mathfrak{B}=L_{p}(X, \mathscr{B}, m)$, $1 \leqq p<\infty$, and $\widetilde{\mathfrak{B}}$ be the set of all $\mathscr{B}$-measurable functions, where two functions are identified if they coincide $m$-almost everywhere. Then, any nonnegative $e$ in $\widetilde{\mathfrak{B}}$ satisfies $(0.1)-(0.3)$. Let $f, g \in \mathfrak{B}$. We have

$$
\begin{aligned}
\varphi_{e}(f, g) & =\int_{X}(f-e)^{+}(x)^{p-1} g(x) m(d x) /\left\|(f-e)^{+}\right\| \|^{p-1} \text { if }(f-e)^{+} \neq 0, \\
& =\left[\int_{\{x: f(x)=e(x)\}} g^{+}(x)^{p} m(d x)\right]^{p-1} \text { if }(f-e)^{+}=0
\end{aligned}
$$

for $1<p<\infty$; and

$$
\varphi_{e}(f, g)=\int_{\{x: f(x)>e(x)\}} g(x) m(d x)+\int_{\{x: f(x)=e(x)\}} g^{+}(x) m(d x)
$$

for $p=1$.

\section{More examples of e-gauge functionals.}

EXAMPLE 3.1. Suppose that a real-valued functional $\psi(f, g)$ defined for $f \in \mathfrak{B}, f \geqq 0$, and $g \in \mathfrak{B}$ satisfies the following:

$$
\text { If } \begin{aligned}
& g \geqq 0 \text { and } f \wedge|h|= 0, \text { then } \psi(f, g-h) \geqq 0 \text { and } \\
& \psi(f, h-g) \leqq 0 ; \\
& \psi(f, g+h) \leqq \\
& \psi g \|+\psi(f, h) ; \\
& \psi(f, \alpha f+g)=\alpha\|f\|+\psi(f, g),
\end{aligned}
$$

$\alpha$ real .

Let $e$ be an element of $\widetilde{\mathfrak{B}}$ satisfying $(0.1)$ and define $\psi_{e}$ by

$$
\psi_{e}(f, g)=\psi\left((f-e)^{+}, g\right), \quad f, g \in \mathfrak{B} .
$$


Then, $\psi_{e}$ is an e-gauge functional. In fact, apply (3.1) with $f, g$, and $h$ replaced by $(f-e)^{+}, \alpha(f \wedge e-g)^{+}$, and $\alpha(g-f \wedge e)^{+}$, respectively, in order to get (0.4). (0.5) follows from (3.2), and (0.6) from (3.3).

REMARK 3.1. If $\psi$ satisfies (3.1)-(3.3), then $\psi^{\prime}$ defined by $\psi^{\prime}(f, g)=-\psi(f,-g)$ satisfies (3.1)-(3.3), too.

EXAMPLE 3.2. Let $[g, f]$ be a functional which satisfies

$$
\begin{gathered}
{[\alpha g+\beta h, f]=\alpha[g, f]+\beta[h, f],} \\
|[g, f]| \leqq\|g\|\|f\|, \quad[f, f]=\|f\|^{2}, \\
{\left[f, f^{+}\right]=\left\|f^{+}\right\|^{2},} \\
{[g, f] \geqq 0 \text { if } f \geqq 0 \text { and } g \geqq 0 .}
\end{gathered}
$$

Let $\sigma$ and $\sigma^{\prime}$ be defined by

$$
\sigma(f, g)=\inf _{|h| \wedge f=0, \beta \geq 0} \tau(f,(g+h) \vee(-\beta f)), \sigma^{\prime}(f, g)=-\sigma(f,-g)
$$

for $f, g \in \mathfrak{B}, f \geqq 0$. Then, any one of the following choices of $\psi$ satisfies (3.1)-(3.3):

$$
\begin{gathered}
\psi(0, g)=0, \psi(f, g)=[g, f] /\|f\| \text { for } f \neq 0, \\
\psi(f, g)=2^{-1}(\tau(f, g)-\tau(f,-g)), \\
\psi(f, g)=\sigma(f, g), \\
\psi(f, g)=\sigma^{\prime}(f, g) .
\end{gathered}
$$

$[g, f]$ is a semi-inner-product used by Phillips [10]. (3.10) is introduced by Hasegawa [5]. $\sigma$ and $\sigma^{\prime}$ are introduced by Sato [11]. The proof for (3.9) is obvious. For the proof of (3.2) and (3.3) with $\psi$ defined by (3.10), use properties of $\tau$ in [5, Proposition 2]. (3.1) is proved from the fact that $f \geqq 0, g \geqq 0$, and $f \wedge|h|=0$ imply $|f-g+h| \leqq$ $|f+g-h|$. For (3.11), use [11, Proposition 3.1]. For (3.12), use Remark 3.1. Our Theorems 1.1 and 1.2 thus include the characterization of $G^{+}(1,0)$ by [10], [5], and [11]. Note that characterization of $G^{+}(1, \gamma)$ is easily obtained from that of $G^{+}(1,0)$, cf. [4]. But there is no favorable relation between $G^{e}(1, \gamma)$ and $G^{e}(1,0)$ in general.

REMARK 3.2. Given $f \geqq 0$ and $g$ in $\mathfrak{B}, \psi(f, g)$ is the same for all $\psi$ satisfying (3.1)-(3.3) if $\|f+\varepsilon g\|$ is differentiable at $\varepsilon=0$. This is a consequence of Example 3.1 and (2.17) combined with Theorem 2.2. Such is the case if $\mathfrak{B}$ is a Hilbert space, where it is easy to see that $\tau(f, g)=\tau^{\prime}(f, g)=(f, g) /\|f\|$ for $f \neq 0$. 
REMARK 3.3. We can characterize $\sigma$ by Phillips' semi-inner-product. Let $f \in \mathfrak{B}, f \geqq 0$, and $f \neq 0$. Let $\Phi_{f}$ be the set of linear functionals $\varphi \in \mathfrak{B}^{*}$ such that $\|\varphi\| \leqq 1, \varphi(f)=\|f\|, \phi \geqq 0$, and $\varphi(g)=$ 0 if $f \wedge|g|=0$. Then we have

$$
\sigma(f, g)=\max _{\varphi \in \Phi_{f}} \varphi(g), \quad \sigma^{\prime}(f, g)=\min _{\varphi \in \Phi_{f}} \varphi(g) .
$$

Hence,

$$
\sigma(f, g)=\max [g, f] /\|f\|, \quad \sigma^{\prime}(f, g)=\min [g, f] /\|f\|,
$$

where maximum and minimum are taken over all $[g, f]$ satisfying (3.5)-(3.7). For the proof, we have only to show the first equation in (3.13), the second being a consequence of the first. If $\varphi \in \Phi_{f}$, we have $\varepsilon^{-1}(\|f+\varepsilon g\|-\|f\|) \geqq \varepsilon^{-1}(\varphi(f+\varepsilon g)-\varphi(f))=\varphi(g)$ and, hence, $\tau(f, g) \geqq \varphi(g)$. Thus

$$
\tau(f,(g+h) \vee(-\beta f)) \geqq \varphi((g+h) \vee(-\beta f)) \geqq \varphi(g+h)=\varphi(g)
$$

if $|h| \wedge f=0$ and $\beta \geqq 0$. Hence $\sigma(f, g) \geqq \varphi(g)$. On the other hand, let us show the existence of $\varphi \in \Phi_{f}$ such that $\varphi(g)=\sigma(f, g)$ for given $g$. We will freely use the properties of $\sigma$ in [11, Proposition 3.1]. Let $\mathfrak{M}_{f, g}$ be the set of $k$ such that $k=\alpha(f+g)+h$ for some $\alpha$ and $h$ such that $|h| \wedge f=0$. Let $\varphi(k)=\alpha \sigma(f, f+g)$ for such $k$. $\varphi(k)$ is uniquely defined and satisfies $\varphi(k) \leqq \sigma(f, k)$ :

$$
\begin{array}{lr}
\varphi(k)=\sigma(f, \alpha(f+g))=\sigma(f, k) & \text { if } \alpha \geqq 0 \\
\varphi(k)=-\sigma(f,-\alpha(f+g)) \leqq \sigma(f, \alpha(f+g))=\sigma(f, k) & \text { if } \alpha<0 .
\end{array}
$$

It can be proved that $\mathfrak{M}_{f, g}$ is a closed subspace. Therefore, noting that $\sigma(f, l)$ is subadditive in $l$ and satisfies $\sigma(f, \alpha l)=\alpha \sigma(f, l), \alpha \geqq 0$, we can extend $\varphi$ to a linear functional on $\mathfrak{B}$ satisfying $\varphi(l) \leqq \sigma(f, l)$ for all $l \in \mathfrak{B}$ by Hahn-Banach theorem. We have $\|\varphi\| \leqq 1$ because $\varphi(l) \leqq \sigma(f, l) \leqq\left\|l^{+}\right\| \leqq\|l\|$ and $-\varphi(l) \leqq \sigma(f,-l) \leqq\left\|l^{-}\right\| \leqq\|l\|$. Since $\varphi(f)+\varphi(g)=\sigma(f, f+g)=\|f\|+\sigma(f, g), \quad \varphi(f) \leqq\|f\|, \quad$ and $\varphi(g) \leqq \sigma(f, g)$, we have $\varphi(f)=\|f\|$ and $\varphi(g)=\sigma(f, g)$. If $l \geqq 0$, then $-\varphi(l) \leqq \sigma(f,-l) \leqq 0$. Thus $\varphi$ belongs to $\Phi_{f}$ and the proof is complete.

REMARK 3.4. For any real or complex Banach space $\mathfrak{B}$, the corresponding theorems for $\boldsymbol{G}(1, \gamma)$ are simpler than those for $\boldsymbol{G}^{e}(1, \gamma)$, and are mostly known. Let $\psi$ be a real functional such that

$$
\tau^{\prime}(f, g) \leqq \psi(f, g) \leqq \tau(f, g) .
$$

Call an operator $A(\psi, \gamma)$-dissipative if $\psi(f, A f) \leqq \gamma\|f\|$. Then, Theorems 1.1 and 1.2 remain valid if we replace $\left(\psi_{e}, \gamma\right)$-dispersiveness 
and $\boldsymbol{G}^{e}(1, \gamma)$ by $(\psi, \gamma)$-dissipativeness and $\boldsymbol{G}(1, \gamma)$. For the proof, we need only note that this is true for $\psi=\tau$ or $\tau^{\prime}$ by $[5,11,4]$. A sufficient condition for (3.15) is that $\psi$ satisfies

$$
\begin{gathered}
-\|g\| \leqq \psi(f, g) \leqq\|g\|, \\
\psi(f, \alpha f+g)=\alpha\|f\|+\psi(f, g), \quad \alpha \text { real } .
\end{gathered}
$$

An operator $A$ is $\left(\tau^{\prime}, \gamma\right)$-dissipative if and only if $(\alpha-\gamma)\|f\| \leqq$ $\|\alpha f-A f\|$ for all $f \in \mathfrak{D}(A)$ and large real $\alpha$. Letting $\Phi_{f}$ be the set of $\varphi \in \mathfrak{B}^{*}$ such that $\|\varphi\| \leqq 1$ and $\varphi(f)=\|f\|$, we have

$$
\tau(f, g)=\max _{\varphi \in \Phi_{f}} \mathscr{R} \varphi(g), \quad \tau^{\prime}(f, g)=\min _{\varphi \in \Phi_{f}} \mathscr{R} \varphi(g), \text { for } f \neq 0 .
$$

Hence $\tau(f, g)$ and $\tau^{\prime}(f, g)$ are the maximum and the minimum, respectively, of $[g, f] /\|f\|$ over all semi-inner-products, that is, functionals satisfying (3.5). This is a consequence of [3, Th. V.9.5]. Conditions for $\tau^{\prime}(f, g)=\tau(f, g), f \neq 0$, are studied by R. C. James [6]. He proves, among others, that $\tau^{\prime}(f, g)=\tau(f, g)$ for all $f \neq 0$ and $g$ if $\mathfrak{B}^{*}$ is strictly convex.

4. Closability and related properties. The following theorem covers all the previous closability results for dissipative and dispersive operators [9, Lemma $3.3 ; 5$, Proposition 7 ; 11, Th. 3].

THEOREM 4.1 Let $A$ be a densely defined linear operator in a real or complex Banach space. If there exist real numbers $M, \gamma$, and $\alpha_{0}$ such that

$$
(\alpha-\gamma)\|f\| \leqq M\|\alpha f-A f\| \text { for } \quad \alpha>\alpha_{0}, f \in \mathfrak{D}(A),
$$

then $A$ is closable and (4.1) holds with $A$ replaced by $\bar{A}$.

Proof. We may assume $M \geqq 1$ and $\alpha_{0} \geqq 0$. It suffices to show that $f_{n} \rightarrow 0$ and $A f_{n} \rightarrow g \neq 0$ produce a contradiction. We may assume $\|g\|=1$. Pick an element $h \in \mathfrak{D}(A)$ such that $\|h-g\|<(3 M)^{-1}$. We have $\|h\|>1-(3 M)^{-1} \geqq 2 / 3$. Let

$$
\varphi_{\alpha, n}=\left\|\alpha f_{n}+h\right\|-M\left\|\alpha f_{n}+h-g-\alpha^{-1} A h\right\| \text { for } \alpha>\alpha_{0} .
$$

We have

$$
\begin{aligned}
& \liminf _{n \rightarrow \infty} \varphi_{\alpha, n} \\
\geqq & \lim _{n \rightarrow \infty}\left(\left\|\alpha f_{n}+h\right\|\right)-M\left\|\alpha f_{n}\right\|-M\|h-g\|-M \alpha^{-1}\|A h\| \\
& >3^{-1}-M \alpha^{-1}\|A h\|
\end{aligned}
$$

on the one hand, and 


$$
\begin{aligned}
\varphi_{\alpha, n} & \leqq\left\|\alpha f_{n}+h\right\|-M\left\|\alpha f_{n}+h-A\left(f_{n}+\alpha^{-1} h\right)\right\|+M\left\|A f_{n}-g\right\| \\
& \leqq \gamma\left\|f_{n}+\alpha^{-1} h\right\|+M\left\|A f_{n}-g\right\|,
\end{aligned}
$$

hence $\lim \sup _{n \rightarrow \infty} \varphi_{\alpha, n} \leqq \gamma \alpha^{-1}\|\mathrm{~h}\|$ on the other. This is a contradiction when $\alpha$ is large. It is obvious that $\bar{A}$ satisfies (4.1).

REMARK 4.1. Let $e$ satisfy (0.1)-(0.3) and $\psi_{e}$ be an e-gauge functional. Let $A$ be a linear densely defined $\left(\psi_{e}, \gamma\right)$-dispersive operator. Then, $A$ is closable and $\bar{A}$ is $\left(\phi_{e}^{\prime}, \gamma\right)$-dispersive. If $\Re(\alpha-A)$ is dense for some $\alpha>\gamma, \bar{A}$ belongs to $G^{e}(1, \gamma)$. In fact, $A$ is closable by Lemma 1.2 and Theorem 4.1 , and $\bar{A}$ is $\left(\varphi_{e}^{\prime}, \gamma\right)$-dispersive by Remark 2.2. If $\mathfrak{R}(\alpha-A)$ is dense, we have $\mathfrak{R}(\alpha-\bar{A})=\mathfrak{B}$ by using (1.5), and hence $A \in G^{e}(1, \gamma)$ by Theorem 1.2. In order that $\Re(\alpha-A)$ be dense for $\alpha>\gamma$, it suffices that $\alpha-A^{*}$ is one-to-one, and hence, $\left(\phi_{0}^{\prime}, \gamma^{\prime}\right)$-dispersiveness or $\left(\tau^{\prime}, \gamma^{\prime}\right)$-dissipativeness of $A^{*}$ for some $\gamma^{\prime}$ suffices.

5. Relation between dispersiveness and dissipativeness. If $A$ is bounded with $\mathfrak{D}(A)=\mathfrak{B}$, or, more generally, if $A$ belongs to $\boldsymbol{G}$, then $\left(\varphi_{0}^{\prime}, \gamma\right)$-dispersiveness of $A$ implies its $\left(\tau^{\prime}, \gamma\right)$-dissipativeness (Theorem 1.2 and Remark 3.4). The same is true if $\Re(\alpha-A)$ is a sublattice for every large $\alpha$ (Lemma 1.4). But we do not know whether this is true in general. Here we restrict our attention to the case where the following condition is satisfied ${ }^{1}$ :

$$
\text { If }\left\|f^{+}\right\|=\left\|g^{+}\right\| \text {and }\left\|f^{-}\right\|=\left\|g^{-}\right\| \text {, then }\|f\|=\|g\| \text {. }
$$

This is essentially the condition considered by F. Bohnenblust [2].

Lemma 5.1. Assume (5.1). If $\left\|f^{+}\right\| \leqq\left\|g^{+}\right\|$and $\left\|f^{-}\right\| \leqq\left\|g^{-}\right\|$, then $\|f\| \leqq\|g\|$.

Proof. We have $\left\|f^{+}\right\|=\alpha\left\|g^{+}\right\|$and $\left\|f^{-}\right\|=\beta\left\|g^{-}\right\|$for some $0 \leqq \alpha \leqq 1$ and $0 \leqq \beta \leqq 1$. Let $h=\alpha g^{+}-\beta g^{-}$. Then, $h^{+}=\alpha g^{+}$and $h^{-}=\beta g^{-}$, and hence $\|h\|=\|f\|$ by the condition (5.1). On the other hand, we have $\|h\| \leqq\|g\|$ since $|h|=\alpha g^{+}+\beta g^{-} \leqq g^{+}+g^{-}=|g|$.

THEOREM 5.1. Assume that $\mathfrak{B}$ satisfies the condition (5.1). Then,

$$
\sup _{f \neq 0, f \in \mathscr{D}_{(A)}} \frac{\tau^{\prime}(f, A f)}{\|f\|} \leqq \sup _{f^{+} \neq 0, f \in \mathfrak{D}^{(A)}} \frac{\varphi_{0}^{\prime}(f, A f)}{\left\|f^{+}\right\|}
$$

${ }^{1}$ All familiar Banach lattices $\left(C, L_{p}, 1 \leqq p \leqq \infty\right.$, etc.) satisfy this condition. As an example of a Banach lattice which does not satisfy the condition, consider the direct sum of $L_{p_{1}}$ and $L_{p_{2}}, p_{1} \neq p_{2}$ with $\left\|f_{1} \oplus f_{2}\right\|=\left\|f_{1}\right\|+\left\|f_{2}\right\|$ and define $f_{1} \oplus f_{2} \leqq$ $g_{1} \oplus g_{2}$ if and only if $f_{1} \leqq g_{1}$ and $f_{2} \leqq g_{2}$. 
for every linear operator $A$. That is, linear $\left(\varphi_{0}^{\prime}, \gamma\right)$-dispersiveness implies $\left(\tau^{\prime}, \gamma\right)$-dissipativeness.

Proof. Suppose the right-hand side in (5.2) is finite and denote it by $\gamma$. Then we have (1.4) and $(\alpha-\gamma)\left\|f^{-}\right\| \leqq\left\|(\alpha f-A f)^{-}\right\|$. Hence, $(\alpha-\gamma)\|f\| \leqq\|\alpha f-A f\|$ by Lemma 5.1. It follows that $\tau^{\prime}(f, A f) \leqq \gamma\|f\|$ for all $f \in \mathfrak{D}(A)$.

6. Infinitesimal generators of nonnegative semigroups. Characterization of the operators in $\boldsymbol{G}^{+}$is an interesting open problem. Here we present some results concerning this problem.

Theorem 6.1. Suppose that $A$ belongs to $\boldsymbol{G}^{+}$. Then,

$$
\sigma(g,-A f) \leqq 0 \text { if } f \in \mathfrak{D}(A), f \geqq 0, g \geqq 0 \text {, and } \sigma(g, f)=0 \text {. }
$$

Proof. Using the properties of $\sigma$ in [11, Proposition 3.1], we have $\sigma\left(g, t^{-1}\left(f-T_{t} f\right)\right) \leqq \sigma\left(g, t^{-1} f\right)+\sigma\left(g,-t^{-1} T_{t} f\right)=\sigma\left(g,-t^{-1} T_{t} f\right) \leqq 0$ and hence $\sigma(g,-A f) \leqq 0$.

THEOREM 6.2. Let $A$ be a bounded linear operator with $\mathfrak{D}(A)=$ $\mathfrak{B}$ and suppose that

$$
\sigma(g, A f) \geqq 0 \text { if } f \in \mathfrak{D}(A), f \geqq 0, g \geqq 0 \text {, and } \sigma(g, f)=0 .
$$

Then, $A \in G^{+}$.

Note that (6.2) is weaker than (6.1), since $\sigma(g, A f) \geqq-\sigma(g,-A f)$.

Proof. For each $f$ we have $\sigma\left(f^{+}, A f^{-}\right) \geqq 0$ and hence

$$
\begin{aligned}
-\sigma\left(f^{+},-A f\right) & \leqq \sigma\left(f^{+}, A f^{+}\right)-\sigma\left(f^{+}, A f^{-}\right) \\
& \leqq \sigma\left(f^{+}, A f^{+}\right) \leqq\left\|A f^{+}\right\| \leqq\|A\|\left\|f^{+}\right\| .
\end{aligned}
$$

Therefore, $A \in G^{+}(1,\|A\|)$.

THeOREM 6.3. If $\mathfrak{B}$ is the space $C(X)$ of continuous functions on a compact space, then any operator in $\boldsymbol{G}$ which satisfies the condition (6.2) belongs to $\boldsymbol{G}^{+}$.

Proof. The resolvent $G_{\alpha}=(\alpha-A)^{-1}$ exists for large $\alpha$, say, $\alpha>\gamma$. It suffices to prove $G_{\alpha} f \geqq 0$ for $f \geqq 0$. We may assume $f(x)>0$ on $X$, since general nonnegative $f$ is approximated by $f+\varepsilon$. Suppose that $G_{\alpha_{0}} f\left(x_{0}\right)<0$ for some $\alpha_{0}>\gamma$ and $x_{0} \in X$. Let $\alpha_{1}$ be the supremum of $\alpha$ such that $G_{\alpha} f(x)<0$ for some $x \in X . \alpha_{1}$ is finite 
because $\alpha G_{\alpha} f \rightarrow f$ as $\alpha \rightarrow \infty$ and $\inf _{x \in X} f(x)>0$. Choose $\beta_{n}$ and $y_{n}$ such that $\beta_{n}$ increases to $\alpha_{1}$ and $G_{\beta_{n}} f\left(y_{n}\right)<0$. Taking a subsequence if necessary, we can find a point $x_{1}$ such that $G_{\alpha_{1}} f\left(y_{n}\right) \rightarrow G_{\alpha_{1}} f\left(x_{1}\right)$. Since $G_{\alpha} f$ is strongly continuous with respect to $\alpha$ by the resolvent equation, $G_{\beta_{n}} f\left(y_{n}\right)$ tends to $G_{\alpha_{1}} f\left(x_{1}\right)$. Hence $G_{\alpha_{1}} f\left(x_{1}\right) \leqq 0$. Since $G_{\alpha_{1}} f \geqq$ 0 by the definition of $\alpha_{1}$, we have $G_{\alpha_{1}} f\left(x_{1}\right)=0$. Let

$$
g(x)=\left\|G_{\alpha_{1}} f\right\|-G_{\alpha_{1}} f(x) .
$$

Using an explicit form of $\sigma[11,6.1]$, we have $\sigma\left(g, G_{\alpha_{1}} f\right)=0$, and hence, by the condition (6.2), $0 \leqq \sigma\left(g, A G_{\alpha_{1}} f\right)=\max A G_{\alpha_{1}} f(x)$, where the maximum is taken over the set of $x$ such that $g(x)=\|g\|$. Thus we can find a point $x_{2}$ such that $G_{\alpha_{1}} f\left(x_{2}\right)=0$ and $A G_{\alpha_{1}} f\left(x_{2}\right) \geqq 0$. Hence $f\left(x_{2}\right)=\alpha_{1} G_{\alpha_{1}} f\left(x_{2}\right)-A G_{\alpha_{1}} f\left(x_{2}\right) \leqq 0$, which is absurd. The proof of Theorem 6.3 is complete.

Added in proof. The results [8] have appeared in the following paper: H. Kunita, Sub-Markov semi-groups in Banach lattices, Proceedings of the International Conference on Functional Analysis and Related Topics, University of Tokyo Press, Tokyo, 1970, 332-343.

\section{REFERENCES}

1. G. Birkhoff, Lattice theory, 3rd ed., Amer. Math. Soc. Coll. Pub. 25, Providence, 1967.

2. F. Bohnenblust, An axiomatic characterization of $L_{p}$-spaces, Duke Math. 6 (1940), 627-640.

3. N. Dunford and J. T. Schwartz, Linear operators, Part I, Interscience, New York, 1958.

4. K. Gustafson and K. Sato, Some perturbation theorems for nonnegative contraction semigroups, J. Math. Soc. Japan, 21 (1969), 200-204.

5. M. Hasegawa, On contraction semi-groups and (di)-operators, J. Math. Soc. Japan 18 (1966), 290-302.

6. R. C. James, Orthogonality and linear functionals in normed linear spaces, Trans. Amer. Math. Soc. 61 (1947), 265-292.

7. Y. Kōmura, Nonlinear semi-groups in Hilbert space, J. Math. Soc. Japan 19 (1967), 493-507.

8. H. Kunita, Sub-Markov semi-groups in Banach lattices, Researches in Mathematical Sciences, No. 57 (Kyôto, 1968), 1-23 (Japanese).

9. G. Lumer and R. S. Phillips, Dissipative operators in a Banach space, Pacific J. Math. 11 (1961), 679-698.

10. R. S. Phillips, Semi-groups of positive contraction operators, Czechoslovak Math. J. (87) 12 (1962), 294-313.

11. K. Sato, On the generators of non-negative contraction semi-groups in Banach lattices, J. Math. Soc. Japan 20 (1968), 423-436.

12. K. Yosida, Functional analysis, Springer, Berlin-Heidelberg-New York, 1965.

Received May 19, 1969. The author is visiting the University of Illinois, and is supported in part by the National Science Foundation. 



\section{PACIFIC JOURNAL OF MATHEMATICS}

\section{EDITORS}

H. SAMelson

Stanford University

Stanford, California 94305

\section{Richard PIERCE}

University of Washington

Seattle, Washington 98105
J. DugundjI

Department of Mathematics

University of Southern California

Los Angeles, California 90007

BASIL GoRDON*

University of California

Los Angeles, California 90024

\section{ASSOCIATE EDITORS}
E. F. BECKENBACH
B. H. NeumanN
F. WOLE
K. YosHIDA

\section{SUPPORTING INSTITUTIONS}

UNIVERSITY OF BRITISH COLUMBIA

CALIFORNIA INSTITUTE OF TECHNOLOGY

UNIVERSITY OF CALIFORNIA

MONTANA STATE UNIVERSITY

UNIVERSITY OF NEVADA

NEW MEXICO STATE UNIVERSITY

OREGON STATE UNIVERSITY

UNIVERSITY OF OREGON

OSAKA UNIVERSITY

UNIVERSITY OF SOUTHERN CALIFORNIA

\author{
STANFORD UNIVERSITY \\ UNIVERSITY OF TOKYO \\ UNIVERSITY OF UTAH \\ WASHINGTON STATE UNIVERSITY \\ UNIVERSITY OF WASHINGTON \\ * * * * \\ AMERICAN MATHEMATICAL SOCIETY \\ CHEVRON RESEARCH CORPORATION \\ TRW SYSTEMS \\ NAVAL WEAPONS CENTER
}

The Supporting Institutions listed above contribute to the cost of publication of this Journal, but they are not owners or publishers and have no responsibility for its content or policies.

Mathematical papers intended for publication in the Pacific Journal of Mathematics should be in typed form or offset-reproduced, (not dittoed), double spaced with large margins. Underline Greek letters in red, German in green, and script in blue. The first paragraph or two must be capable of being used separately as a synopsis of the entire paper. The editorial "we" must not be used in the synopsis, and items of the bibliography should not be cited there unless absolutely necessary, in which case they must be identified by author and Journal, rather than by item number. Manuscripts, in duplicate if possible, may be sent to any one of the four editors. Please classify according to the scheme of Math. Rev. 36, 1539-1546. All other communications to the editors should be addressed to the managing editor, Richard Arens, University of California, Los Angeles, California, 90024.

50 reprints are provided free for each article; additional copies may be obtained at cost in multiples of 50 .

The Pacific Journal of Mathematics is published monthly. Effective with Volume 16 the price per volume (3 numbers) is $\$ 8.00$; single issues, $\$ 3.00$. Special price for current issues to individual faculty members of supporting institutions and to individual members of the American Mathematical Society: $\$ 4.00$ per volume; single issues $\$ 1.50$. Back numbers are available.

Subscriptions, orders for back numbers, and changes of address should be sent to Pacific Journal of Mathematics, 103 Highland Boulevard, Berkeley, California, 94708.

PUBLISHED BY PACIFIC JOURNAL OF MATHEMATICS, A NON-PROFIT CORPORATION

Printed at Kokusai Bunken Insatsusha (International Academic Printing Co., Ltd.), 7-17, Fujimi 2-chome, Chiyoda-ku, Tokyo, Japan.

* Acting Managing Editor. 


\section{Pacific Journal of Mathematics}

\section{Vol. 33, No. $2 \quad$ April, 1970}

Raymond Balbes and Alfred Horn, Projective distributive lattices ....... 273

John Findley Berglund, On extending almost periodic functions ......... 281

Günter Krause, Admissible modules and a characterization of reduced left

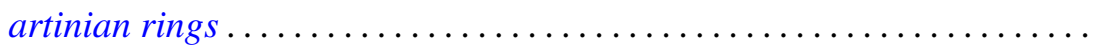

Edward Milton Landesman and Alan Cecil Lazer, Linear eigenvalues and a nonlinear boundary value problem ....................... 311

Anthony To-Ming Lau, Extremely amenable algebras ............... 329

Aldo Joram Lazar, Sections and subsets of simplexes .............. 337

Vincent Mancuso, Mesocompactness and related properties ............. 345

Edwin Leroy Marsden, Jr., The commutator and solvability in a generalized orthomodular lattice .................................. 357

Shozo Matsuura, Bergman kernel functions and the three types of canonical domains.......................................... 363

S. Mukhoti, Theorems on Cesàro summability of series .............. 385

Ngô Van Quê, Classes de Chern et théorème de Gauss-Bonnet ........... 393

Ralph Tyrrell Rockafellar, Generalized Hamiltonian equations for convex problems of Lagrange ................................ 411

Ken iti Sato, On dispersive operators in Banach lattices ............. 429

Charles Andrew Swanson, Comparison theorems for elliptic differential

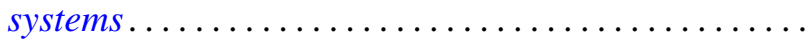

John Griggs Thompson, Nonsolvable finite groups all of whose local subgroups are solvable. II

David J. Winter, Cartan subalgebras of a Lie algebra and its ideals ... 